\title{
Assessment of Tubal Patency with Tridimensional Coded Contrast Imaging Hysterosalpingo-Contrast- Sonography Compared with Conventional HyCoSy: A Preliminary Study
}

\author{
${ }^{1}$ Juan Luis Alcázar, ${ }^{2}$ Manuela Moya, ${ }^{3}$ Maria Caparros, ${ }^{4}$ Aina Salas, ${ }^{5}$ Tania Errasti, ${ }^{6}$ Alvaro Ruiz-Zambrana
}

\begin{abstract}
Objective: The aim of this preliminary study is to analyze and compare tridimensional coded contrast imaging hysterosalpingocontrast-sonography (3D-CCl HyCoSy) with conventional hysterosalpingo-contrast-sonography (HyCoSy) with saline serum for the evaluation of tubal patency in women with sterility.
\end{abstract}

Materials and methods: This was a prospective preliminary observational study performed in the Clinica Universidad de Navarra. Intending to compare 3D-CCl HyCoSy with conventional HyCoSy, we decided and perform conventional HyCoSy between 1st of January 2015 and 15th of February 2015 and 3D-CCl HyCoSy between 16th of February and 15th of April 2015, in different sets of patients. Exclusion criteria were: recent vaginal infection, previous pelvic inflammatory disease, previous salpingectomy or hidrosalpinx diagnosed previously or while performing the ultrasound examination.

Results: Total number of cases was 22 (12 women underwent conventional HyCoSy and 10 a 3D-CCl HyCoSy). The mean age of patients was 33.8 years (SD: 3.2 range: $23-40$ years). Three cases $(100 \%)$ of Fallopian tube obstruction were detected by 3D-CCI HyCoSy and confirmed by laparoscopic tubal chromopertubation (LTC). Four of the six cases (67\%) of obstruction detected by conventional HyCoSy were confirmed by hysterosalpingography/LTC (HSG/LTC). Two cases of severe post-procedure pain were reported in women who underwent conventional HyCoSy.

Conclusion: The results of the study show that 3D-CCI HyCoSy was better to visualize the entire Fallopian tube and evaluate its patency.

Keywords: Hysterosalpingography, Tridimensional coded contrast imaging hysterosalpingo-contrast-sonograph, Tubal chromoperturbation, Tubal patency.

How to cite this article: Alcázar JL, Moya $M$, Caparros $M$, Salas A, Errasti T, Ruiz-Zambrana A. Assessment of Tubal

\footnotetext{
${ }^{1}$ Full Professor, ${ }^{2-4}$ Resident, ${ }^{5,6}$ Specialist

1,3-6Department of Obstetrics and Gynecology, Clinica Universidad de Navarra, University of Navarra, Pamplona, Spain

${ }^{2}$ Department of Obstetrics and Gynecology, University Hospital Mutua de Terrassa, Terrassa, Spain

Corresponding Author: Juan Luis Alcazar, Full Professor Department of Obstetrics and Gynecology, Clínica Universidad de Navarra, Avenida Pío XII 36, 3110, Pamplona, Spain Phone: +34-948-296234, e-mail: jlalcazar@unav.es
}

Patency with Tridimensional Coded Contrast Imaging Hysterosalpingo-Contrast-Sonography Compared with Conventional HyCoSy: A Preliminary Study. Donald School J Ultrasound Obstet Gynecol 2016;10(1):103-106.

\section{Source of support: Nil}

Conflict of interest: None

\section{INTRODUCTION}

Tubal patency evaluation is one of the basic test performed when there is a problem of infertility in a couple. Classically, the used tests to rule out tubal obstruction are the hysterosalpingography (HSG) and tubal chromoperturbation (CT) at laparoscopy. ${ }^{1}$

Hysterosalpingo-contrast-sonography (HyCoSy) with saline serum or gas as an alternative to HSG/CL was introduced in the nineties. ${ }^{2}$ Several studies has shown that HyCoSy is well-tolerated ${ }^{3-5}$ and have a diagnostic reliability similar to HSG and CL.

However, in contrast to HSG/CT, HyCoSy has some limitations because it is difficult to see the entire trajectory and the moment when the serum or gas spill out the end of the fallopian tube. Consequently, the examiner may hesitate about the tubal permeability. The use of ultrasound contrast (which highlight the return signal) combined with coded contrast imaging ${ }^{6}(\mathrm{CCI})$ could it be a possible solution to that.

In coded contrast imaging (CCI), the ultrasound machine emits a beam at a selected frequency and receives a narrow band of harmonic signal, avoiding overlap between the tissue and the contrast response. The image displayed is based only on harmonics signal produced by contrast-medium microspheres; broadband ultrasonic signals from surrounding tissue are filtered out completely.

Besides, three-dimensional ultrasound could allow for tubal cavity reconstruction, what is named 3D-CCI HyCoSy.

The aim of this preliminary study is to analyze and compare 3D-CCI HyCoSy with conventional HyCoSy with saline serum for the evaluation of tubal patency in women with sterility. 


\section{MATERIALS AND METHODS}

\section{Design}

This was a prospective observational study performed in the Clinica Universidad de Navarra from 1st of January 2015 to 15th of April 2015.

\section{Patients}

All women with primary or secondary sterility managed at our institution during the period described were considered candidates for the study. Patients were informed and they gave their written approval previously to the procedure. To avoid memory bias of the examiner we considered not to perform both procedures to each women. Intending to compare 3D-CCI HyCoSy with conventional HyCoSy, we decided to perform conventional HyCoSy between 1st of January 2015 and 15th of February 2015 and 3D-CCI HyCoSy between 16th of February and 15th of April 2015, in different sets of patients.

Patients with recent vaginal infection, previous pelvic inflammatory disease, previous salpingectomy or hidrosalpinx diagnosed previously or while performing the ultrasound examination were excluded.

\section{Ultrasound Procedure}

We performed to all patients a simple transvaginal ultrasound to study biometrical and morphologic characteristics of the uterus and rule out uterine and adnexal pathology. All ultrasound were done between the 5 th and 7 th day of the menstrual cycle. After this initial evaluation, we performed HyCoSy following the next protocol:

\section{Conventional HyCoSy}

We made a vaginal and cervix evaluation after the insertion of a speculum. We performed antisepsis with Betadine skin prep solution (3\%) and inserted through the cervix an 8 F Foley catheter in the uterus. We filled the Foley catheter balloon with 1 to $2 \mathrm{cc}$ and pulled it out to seal off the internal orifice of the cervix and to avoid saline leaks.We did not use prophylactic antibiotic. Subsequently, we instilled 10 to 20 cc of sterile saline blended with air to create bubbles, which augment the ultrasound contrast. Using power Doppler, we evaluate the permeability of Fallopian tubes observing how the contrast flowed through it. In order to do this, we oriented in the transverse plane the ultrasound probe, to the uterine fundus and laterally (to the uterine corns). Afterwards, the vaginal probe was moved to anexial zones to visualize the ovaries and the pass of saline through the Fallopian tubes (Fig. 1).

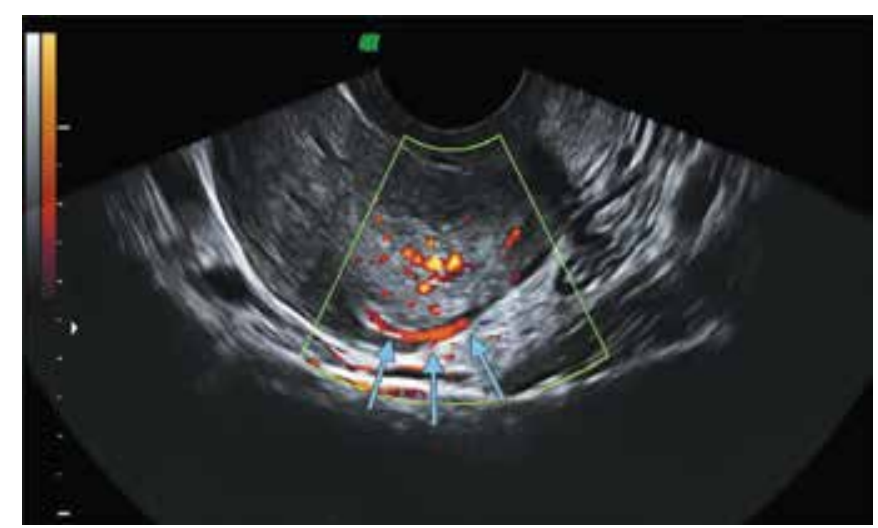

Fig. 1: Hysterosalpingo-contrast-sonography using conventional method. Intramural passage is seen using power Doppler (arrows)

The examiner obtained the following information: (a) tubal permeability (yes/no), visualization of the intramural portion of the Fallopian tube (yes/no), visualization of the fimbrial portion of the Fallopian tube (yes/no), confidence in tubal patency diagnosis (sure/ uncertain).

These procedures were made with Voluson E8 (GE Healthcare, USA), vaginal probe (EC 5-9 MHz) and default factory machine settings for uterine examination (both gray scale and Doppler).

\section{D-CCl HyCoSy}

In order to do this procedure, we performed the same previous catheter insertion in the uterus that we have done in the conventional HyCoSy. Even so, in this case, we used a ultrasonic contrast kit that contains an intracervical catheter, two syringes (one with ultrasonic contrast and another with saline serum), a connector for both syringes to blend the serum with the contrast (ExEm ${ }^{\odot}$, GISKIT BV, Holland). We instilled 5 to $10 \mathrm{cc}$ of the contrast with the same orientation as we did for the conventional HyCoSy. Before instillation, it is necessary to start CCI function. This function dims significantly the screen, but also enhance the contrast for allowing a better visualization of it. As if in the conventional HyCoSy, we moved the ultrasound probe laterally observe how the contrast flowed through the Fallopian tubes and it spill out by the fimbrial portion (Fig. 2). Afterwards, the 3D window was activated when it was properly adjusted to the entire screen, and it was in the transverse plane centrally and deeply enough to include both annexes. Once the volume was obtained, we save it to be analyzed offline. The 3D volume analysis was done using the rendering mode HDLive and the magic-cut to attain the uterine cavity and Fallopian tubes representations (Fig. 3).

As in the conventional HyCoSy, the examiner obtained the following information: (a) tubal permeability (yes/no), visualization of the intramural portion of the Fallopian tube (yes/no), visualization of the fimbrial 


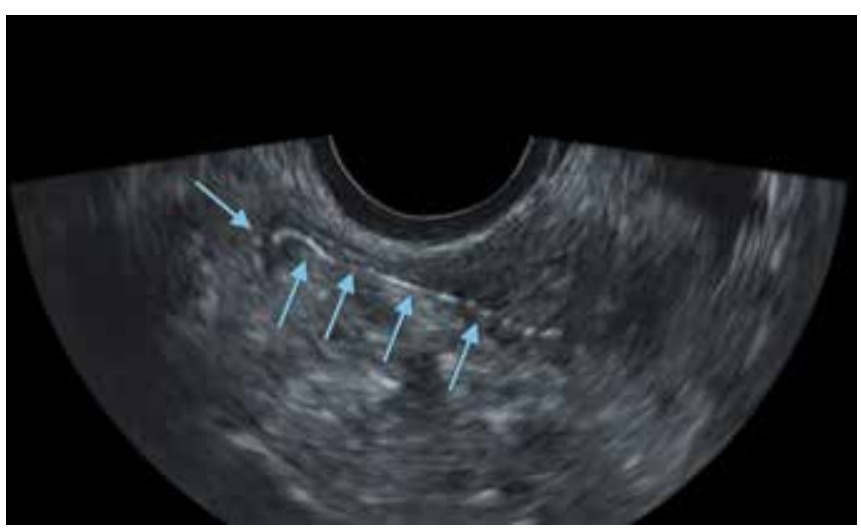

Fig. 2: Hysterosalpingo-contrast-sonography using $\mathrm{CCl}$. The complete tube is seen. Contrast passage depicted as a white line (arrows)

portion of the Fallopian tube (yes/no), confidence in tubal patency diagnosis (sure/uncertain).

To avoid inter-observer variability one examiner conducted all procedures. In case of suspicion of tubal obstruction a HSG or CTL were indicated.

Data are presented as absolute numbers and percentages. Percentages were compared with $\mathrm{Z}$ statistic. A $\mathrm{p}<0.05$ was considered statistically significant. Statistical analysis was performed with Statistical Package for the Social Sciences (SPSS) version 20.0 (SPSS Inc, USA).

\section{RESULTS}

During the study period, 12 women underwent conventional HyCoSy and 10 a 3D-CCI HyCoSy. The total number of Fallopian tubes evaluated were 44 (22 with conventional HyCoSy and 20 with 3D-CCI HyCoSy).

The mean age of patients was 33.8 years (SD: 3.2 range: 23-40 years).

Results that compare both techniques are shown in Table 1.

The three cases (100\%) of Fallopian tube obstruction detected by 3D-CCI HyCoSy were confirmed by LTC. Four of the six cases $(67 \%)$ of obstruction detected by conventional HyCoSy were confirmed by HSG/LTC. With this technique, there were two false positive cases.

Table 1: Results comparing both techniques

\begin{tabular}{lll}
\hline & $\begin{array}{l}\text { Conventional } \\
\text { HyCoSy }\end{array}$ & $3 D-C C l$ HyCoSy \\
\hline Obstruction & $6 / 24(25 \%)$ & $3 / 20(15 \%)$ \\
$\begin{array}{l}\text { Intramural portion } \\
\text { visualization }\end{array}$ & $18 / 18(100 \%)$ & $17 / 17(100 \%)$ \\
$\begin{array}{l}\text { Ampulla portion } \\
\text { visualization }\end{array}$ & $5 / 18(27.8 \%)$ & $16 / 17(94.1 \%)$ \\
$\begin{array}{l}\text { Fimbriae portion } \\
\text { visualization }\end{array}$ & $1 / 18(5.5 \%)$ & $15 / 17(88.2 \%)$ \\
$\begin{array}{l}\text { Diagnostic } \\
\text { confidence }\end{array}$ & $21 / 24(87.5 \%)$ & $20 / 20(100 \%)$ \\
\hline${ }^{*} p<0.05$ & & \\
\hline
\end{tabular}

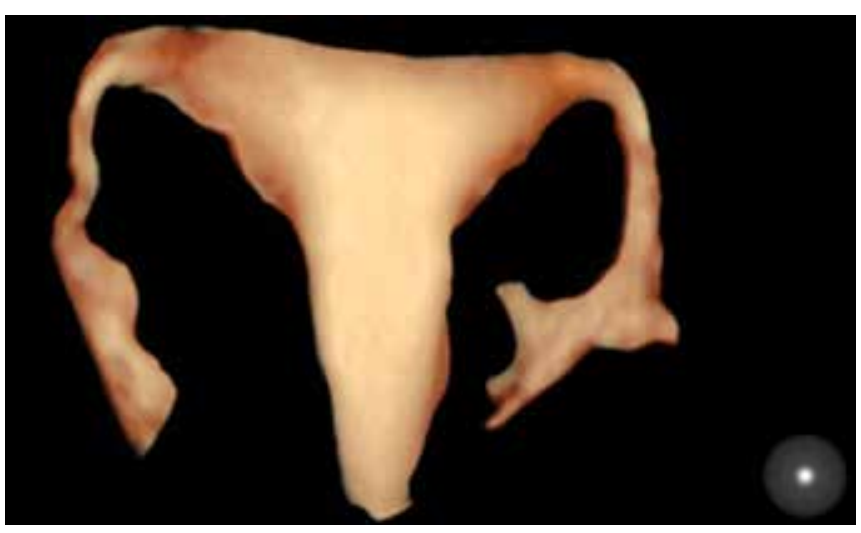

Fig. 3: Three-dimensional reconstruction of the uterine cavity and tubes after 3D-CCl-HyCoSy

Two cases of severe post-procedure pain were reported in women who underwent conventional HyCoSy. Any patient from the group who underwent 3D-CCI HyCoSy referred post-procedure pain.

\section{COMMENT}

In this preliminary study, we compared certain aspects of the technique to visualize the Fallopian tube conventional HyCoSy with 3D-CCI HyCoSy. The results of the study show that 3D-CCI HyCoSy was better to visualize the entire Fallopian tube and evaluate the permeability of it. Besides, the examiner is more confident with 3D-CCI HyCoSy.

The main limitation of our study is the small series. Another limitation is that we did not assess intra- and interobserver reproducibility.

Few studies have assessed the role of 3D-HyCoSy. Kiyokawa et al evaluated 25 women with 3D-HyCoSy using X-ray hysterosalpingography (X-HSG) as reference standard. They reported a sensitivity of $82.6 \%$ and a specificity of $100 \%$, respectively. ${ }^{7}$

Ali et al assessed 50 cases with 3D-HyCoSy and compared with X-HSG. They found that 3D-HyCoSy was a good alternative to X-HSG. Not only for assessing tubal patency but also for depicting uterine cavity. ${ }^{8}$

Chan et al compared 3D-HyCoSy and laparoscopic chromopertubation in 21 infertile women. They reported a sensitivity of $100 \%$ for detecting tubal obstruction, but specificity was as low as $67 \%{ }^{9}$

The largest series using 3D-HyCoSy has been reported by Kupesic et al. They evaluated 116 women and reported a sensitivity of $100 \%$ and specificity of $99 \%$, respectively. ${ }^{10}$

Tridimensional coded contrast imaging hysterosalpingo-contrast-sonography has certain techniques advantages because less quantity of contrast is needed, no Doppler is used (so there is no confusion with vessels), and the contrast persists more time which allows to evaluate calmly and to obtain a 3D volume easily. 
To date only two study have evaluated 3D-CCI HyCoSy. Zhou et al assessed tubal patency in 75 women with infertility by 3D-CCI HyCoSy using laparoscopic chromopertubation as reference test. They reported a sensitivity of $93.5 \%$, specificity of $86.3 \%$, positive and negative predictive values of 87.8 and $92.6 \%$, respectively, and diagnostic accuracy of $90.0 \%{ }^{11}$

Exacoustous et al compared 2D-HyCoSy with 3D-CCI HyCoSy in the same set of patients and found out that agreement between both methods was very high (97\%). ${ }^{6}$

Our results are consistent with these two previous studies. If these results were confirmed with larger studies, 3D-CCI HyCoSy could be established as the first technique recommended in the study of fallopian tubal permeability.

\section{REFERENCES}

1. National Collaborating Centre for Women's and Children's Health (UK). Fertility: Assessment and Treatment for People with Fertility Problems. London: Royal College of Obstetricians and Gynecologists (UK); 2013 Feb.

2. Fleischer AC, Vazquez JM, Cullinan JA, Eisenberg E. Sonohysterography combined with sonosalpingography: correlation with endoscopic findings in infertility patients. J Ultrasound Med 1997;16(6):381-384.

3. Jeanty P, Besnard S, Arnold A, Turner C, Crum P. Air contrast sonohysterography as a first step assessment of tubal patency. J Ultrasound Med 2000;19(8):519-527.

4. Hajishafiha M, Zobairi T, Zanjani VR, Ghasemi-Rad M, Yekta Z, Mladkova N. Diagnostic value of sonohysterography in the determination of the fallopian tube patency as an initial step of routine infertility assessment. J Ultrasound Med 2009;28(12):1671-1677.

5. Marci R, Marcucci I, Marcucci AA, Pacini N, SalaconeP, Sebastianelli A, Caponecchia Lo Monte G, Rago R. Hysterosalpingo contrast sonography: evaluation of the pain, perception, side effects and complications. BMC Med Imaging 2013;13:28.

6. Exacoustous C, Di Giovanni A, Szabolcs B, Romeo V, Romanini ME, Luciano D, Zupi E, Arduini D. Automated three-dimensional coded contrast imaging hysterosalpingocontrast sonography: feasibility in office tubal patency testing. Ultrasound Obstet Gynecol 2013;41(3):328-335.

7. Kiyokawa K, Masuda H, FuyukiT, et al. Three-dimensional hysterosalpingo-contrast sonography as an outpatient procedure to assess infertile women: a pilot study. Ultrasound Obstet Gynecol 2000;16(7):648-654.

8. Ali AM, Kassem MA. Three-dimensional sonohysterography versus standard hysterosalpingography in infertile woman. Middle East Fertil Soc J 2005;10:219-222.

9. Chan $\mathrm{CC}, \mathrm{Ng}$ EH, Tang OS, Chan KK, Ho PC. Comparison of three-dimensional hysterosalpingo-contrast-sonography and diagnostic laparoscopy with chromopertubation in the assessment of tubal patency for the investigation of subfertility. Acta Obstet Gynecol Scand 2005;84(9):909-913.

10. Kupesic S, Plavsic BM. 2D and 3D hysterosalpingo-contrastsonography in the assessment of uterine cavity and tubal patency. Eur J Obstet Gynecol Reprod Biol 2007;133(5): 64-69.

11. Zhou L, Zhang X, Chen X, et al. Value of three-dimensional hysterosalpingo-contrast sonography with SonoVue in the assessment of tubal patency. Ultrasound Obstet Gynecol 2012;40(1):93-98. 